\title{
Malignant Nephrosclerosis in a Patient with Familial Mediterranean Fever
}

\author{
Masayuki Yamanouchi ${ }^{1}$, Yoshifumi Ubara ${ }^{1,2}$, Aya Imafuku ${ }^{1}$, Masahiro Kawada ${ }^{1}$, Mise Koki ${ }^{1}$, \\ Keiichi Sumida ${ }^{1}$, Rikako Hiramatsu ${ }^{1}$, Eiko Hasegawa ${ }^{1}$, Noriko Hayami ${ }^{1}$, Tatsuya Suwabe ${ }^{1}$, \\ Junichi Hoshino ${ }^{1}$, Naoki Sawa ${ }^{1}$, Kenichi Ohashi ${ }^{3}$, Takeshi Fujii ${ }^{3}$, \\ Masayuki Matsuda ${ }^{4}$ and Kenmei Takaichi ${ }^{1,2}$
}

\begin{abstract}
A 37-year-old man was admitted to our hospital for an evaluation of renal dysfunction and hypertension. The C-reactive protein level was $6.0 \mathrm{mg} / \mathrm{dL}$, and the serum renin activity was extremely high. A renal biopsy showed malignant nephrosclerosis-like lesions with an onion skin pattern. He had a history of recurrent abdominal pain associated with periodic fevers above 38 degrees that resolved within three days. A MEditerranean FeVer (MEFV) gene analysis revealed that he was homozygous for the E148Q polymorphism (exon 2) and heterozygous for the L110P polymorphism (exon 2). The present case demonstrates that persistent subclinical inflammation can lead to malignant nephrosclerosis in familial Mediterranean fever patients with this genotype.
\end{abstract}

Key words: MEFV gene, E148Q mutation, malignant nephrosclerosis, familial Mediterranean fever, hemodialysis

(Intern Med 54: 2643-2646, 2015)

(DOI: 10.2169/internalmedicine.54.4937)

\section{Introduction}

Familial Mediterranean fever (FMF) is an autoinflammatory disease caused by a mutation of the MEditerranean FeVer (MEFV) gene. According to the Tel Hashomer criteria, typical episodes of FMF last from 12 hours to three days and feature a fever above 38 degrees accompanied by peritonitis, pleuritis and/or monoarthritis of the hip, knee or ankle joint (1). This disease most commonly occurs in populations of eastern Mediterranean descent, although it has also recently been reported in Japanese individuals (2-5). The pathogenesis of FMF is mediated by inflammatory cytokines. The persistence of inflammation during the intervals between episodes results in kidney involvement by reactive amyloid A (AA) amyloidosis, which is the most severe form of FMF but is limited to patients with specific MEFV gene mutations, such as M694V in eastern Mediterranean peoples and M694I in the Japanese population (2-4). Other types of non-amyloid renal involvement have also been described in patients with FMF $(6,7)$; however, the relationship between FMF and non-amyloid kidney disease is unclear. We encountered a patient with FMF, in whom renal histology demonstrated malignant nephrosclerosis. We herein discuss this case with regard to the association between renovascular disease and autoimmune inflammatory disease.

\section{Case Report}

In March 2011, a 37-year-old Japanese man was admitted to our hospital for an evaluation of recurrent abdominal pain associated with fever and renal dysfunction.

Abdominal pain was first noted at 18 years of age and then became recurrent thereafter, although it always subsided within three days without medication. This pain occurred every three months from 25 years of age. Hyperten-

${ }^{1}$ Nephrology Center, Toranomon Hospital, Japan, ${ }^{2}$ Okinaka Memorial Institute for Medical Research, Toranomon Hospital, Japan, ${ }^{3}$ Department of Pathology, Toranomon Hospital, Japan and ${ }^{4}$ Department of Internal Medicine (Neurology and Rheumatology), Shinshu University, Japan Received for publication January 10, 2015; Accepted for publication February 24, 2015

Correspondence to Dr. Yoshifumi Ubara, ubara@toranomon.gr.jp 


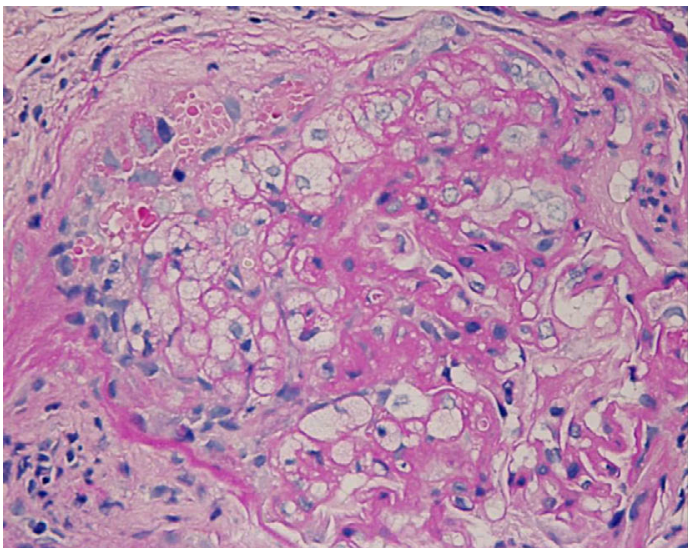

a

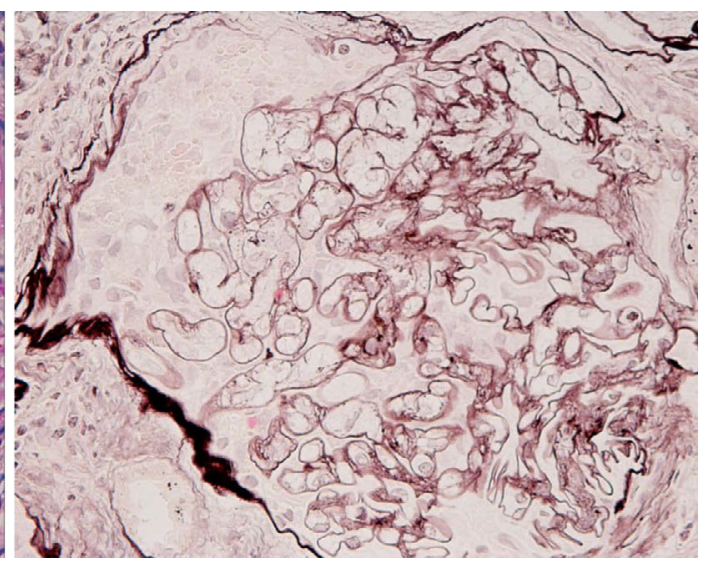

b

Figure 1. Light microscopy shows the collapse of almost all glomeruli, as well as prominent edematous widening of the subendothelial spaces of the glomerular capillary walls. (a) Periodic acid-Schiff stain. (b) Periodic acid methenamine silver stain.

sion (blood pressure: 180/90 $\mathrm{mmHg}$ ) and renal impairment [serum creatinine (Cre): $1.2 \mathrm{mg} / \mathrm{dL}$ ] were detected on an annual screening examination in April 2010; however, no action was taken. In October 2010, he developed severe abdominal pain and a fever lasting for three days, with an elevation of the Cre level to $3.6 \mathrm{mg} / \mathrm{dL}$. The C-reactive protein (CRP) level was also elevated at $6.0 \mathrm{mg} / \mathrm{dL}$, although it subsequently decreased to $2.0 \mathrm{mg} / \mathrm{dL}$. Thereafter, he experienced further episodes of abdominal pain that subsided within three days and were associated with an elevation of the CRP level, indicating intermittent active inflammation during his clinical course.

On admission, the patient was $169 \mathrm{~cm}$ tall and weighed $77 \mathrm{~kg}$. His blood pressure was $175 / 113 \mathrm{mmHg}$ and his temperature was $38.6^{\circ} \mathrm{C}$. No arthropathy was noted. Hematology tests revealed the white blood cell (WBC) count to be $10,000 / \mu \mathrm{L}$, the red blood cell count was $4.69 \times 10^{6} / \mu \mathrm{L}$, the hemoglobin level was $13.0 \mathrm{~g} / \mathrm{dL}$ and the platelet count was $24.6 \times 10^{4} / \mu \mathrm{L}$. Other laboratory parameters were as follows: total protein, $6.8 \mathrm{~g} / \mathrm{dL}$; albumin, $3.5 \mathrm{~g} / \mathrm{dL}$; serum urea nitrogen, $38 \mathrm{mg} / \mathrm{dL}$; serum creatinine, $4.6 \mathrm{mg} / \mathrm{dL}$; total cholesterol, $213 \mathrm{mg} / \mathrm{dL}$; sodium, $143 \mathrm{mmol} / \mathrm{L}$; potassium, 4.3 $\mathrm{mEq} / \mathrm{L}$; chloride, $108 \mathrm{mmol} / \mathrm{L}$; CRP, $6.0 \mathrm{mg} / \mathrm{dL}$; and serum amyloid A protein (SAA), $14.7 \mu \mathrm{g} / \mathrm{mL}$ (normal: <8.0). The IL-1 $\beta$ titer was not measured. Immunological tests revealed an antinuclear antibody titer less than 5.0 (normal: <20.0) and a rheumatoid factor (RF) level of $0 \mathrm{IU} / \mathrm{mL}$ (normal: < 10). An enzyme-linked immunosorbent assay (ELISA) was negative for anti-proteinase-3 (PR-3) anti-neutrophil cytoplasmic antibody (ANCA) and myeloperoxidase ANCA (MPO-ANCA). Anti-glomerular basement membrane antibodies were also negative. The serum C3 level was $122 \mathrm{mg}$ / $\mathrm{dL}$ (normal: $>86 \mathrm{mg} / \mathrm{dL}$ ), the $\mathrm{C} 4$ level was $31 \mathrm{mg} / \mathrm{dL}$ (normal: $>18 \mathrm{mg} / \mathrm{dL}$ ) and the $\mathrm{CH} 50$ level was $58 \mathrm{U} / \mathrm{mL}$ (normal: $>30 \mathrm{U} / \mathrm{mL}$ ). The serum level of $\mathrm{IgG}$ was $1,028 \mathrm{mg} / \mathrm{dL}$, while that of $\operatorname{IgA}$ was $147 \mathrm{mg} / \mathrm{dL}$ and that of $\operatorname{IgM}$ was 81.2 $\mathrm{mg} / \mathrm{dL}$. The serum renin activity was extremely high at 20.0 $\mathrm{ng} / \mathrm{mL} / \mathrm{hr}$ (normal range: 0.3 to 2.9 ) and the aldosterone level was elevated at $23.8 \mathrm{ng} / \mathrm{mL}$ (normal range: 2.99 to 15.9). The adrenocorticotropic hormone (ACTH) level was $5.2 \mathrm{pg} / \mathrm{mL}$ (normal range: 7.2-62.3), the cortisol level was $1.4 \mu \mathrm{g} / \mathrm{dL}$ (normal range: 4.5-21.1), the adrenaline level was $0.03 \mathrm{ng} / \mathrm{mL}$ (normal range: $<0.1$ ) and the dopamine level was $<0.01 \mathrm{ng} / \mathrm{nL}$ (normal range: $<0.02$ ). The urine sediment contained $<1$ erythrocytes per high-power field, the 24-hour protein excretion was $6.2 \mathrm{~g}$ and the creatinine clearance was $23.1 \mathrm{~mL} / \mathrm{min}$.

Gastrointestinal endoscopy and abdominal ultrasonography revealed no abnormal findings. Magnetic resonance (MR) angiography found no evidence of renal artery stenosis, although the patient's kidneys were slightly small, with a long axis of $10 \mathrm{~cm}$ bilaterally. Echocardiography showed diffuse left ventricular hypertrophy with a left ventricular mass (LVM) estimated to weigh $501 \mathrm{~g}$ (normal range: 148 \pm $26 \mathrm{~g}$ ). Fundoscopy showed papilledema with swelling of the optic disc and blurred margins.

\section{Renal biopsy}

A renal biopsy was performed to investigate the kidney disease. Light microscopy of a renal biopsy specimen containing 56 glomeruli revealed global sclerosis in three glomeruli. Almost all of the glomeruli showed collapse, and there was prominent edematous widening of the subendothelial spaces in the glomerular capillary walls (Fig. 1). Concentric subendothelial edematous thickening of the arterioles (onion skin pattern) with luminal narrowing or obstruction was prominent; however, no fibrinoid necrosis of the small arteries or arterioles was evident (Fig. 2). Moderate to severe tubulointerstitial fibrosis with edema was prominent, although cholesterol crystals and vasculitis were not noted. The renal tissue specimen was negative for Congo-red staining, while immunofluorescence was negative for $\operatorname{IgG}, \operatorname{IgA}$, $\operatorname{IgM}$ and $\mathrm{C} 3$. Electron microscopy revealed prominent wrinkling of the glomerular capillary walls, without electron- 

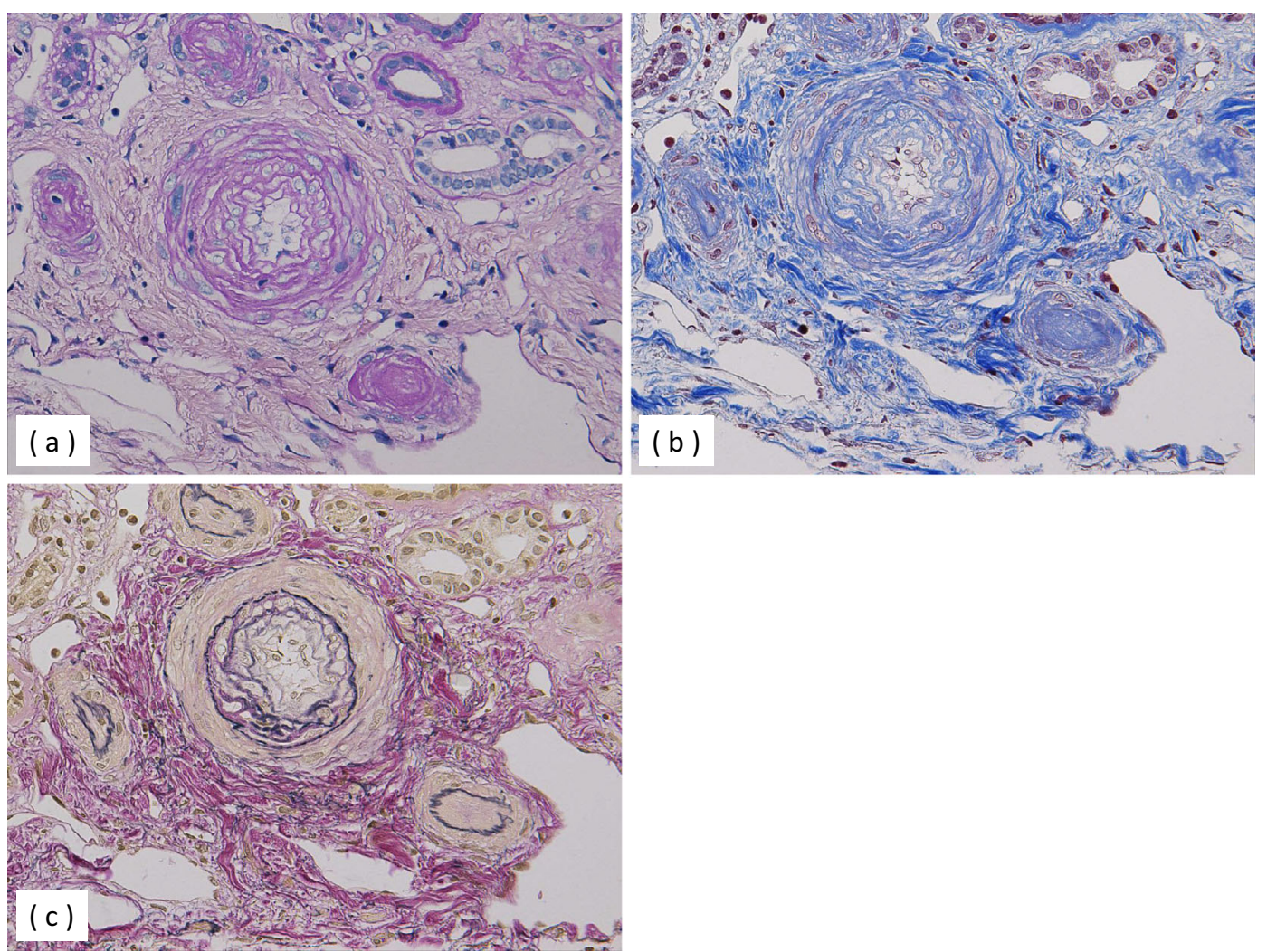

Figure 2. Light microscopy shows concentric subendothelial edematous thickening of the arterioles (onion skin pattern) with narrowing or obstruction of the vascular lumen. (a) Periodic acid-Schiff stain. (b) Masson trichrome stain. (c) Elastica Masson stain.

dense deposits. These histological findings were consistent with a diagnosis of malignant nephrosclerosis according to a previous report (8), although no fibrinoid necrosis was observed.

An endoscopic biopsy of the colon, stomach and duodenum was negative for Congo-red staining.

\section{MEFV gene analysis}

An analysis of the MEFV gene was performed because the patient had a history of recurrent abdominal pain associated with periodic fevers, although AA amyloidosis was negative. After obtaining informed consent, an assessment of all exons of the MEFV gene (1 to 10) was performed using direct sequencing according to the previously reported method (9). This analysis confirmed that the patient was homozygous for the marenostrin/pyrin variant E148Q polymorphism (exon 2) and heterozygous for the L110P polymorphism (exon 2). Therefore, the diagnosis of familial Mediterranean fever was confirmed. His two sons were found to be heterozygous for the E148Q polymorphism; however, they have so far remained asymptomatic.

\section{Clinical course}

Treatment with colchicine $(0.5 \mathrm{mg} /$ day $)$ was started, as well as intensive antihypertensive therapy using an angiotensin-converting enzyme inhibitor and a dihydropyridine calcium channel blocker. His abdominal pain subsided and the CRP level declined to $1.0 \mathrm{mg} / \mathrm{dL}$; however, the renal dysfunction progressed. In January 2012, hemodialysis was initiated because of congestive heart failure.

\section{Discussion}

Said et al. reviewed the renal biopsy findings of 15 patients with a long history of FMF (6) and reported that seven patients had amyloidosis, six patients had mesangial proliferative glomerulonephritis (MsPGN) and two patients had rapidly progressive glomerulonephritis (RPGN). Among the six patients with MsPGN, immunofluorescence was positive for mesangial $\operatorname{IgA}$ deposits (IgA nephropathy) in three patients and $\operatorname{IgM}$ mesangial deposits in three patients (IgM nephropathy). The authors concluded that IgA nephropathy, IgM nephropathy and RPGN should be considered to be non-amyloid renal diseases that can occur in patients with FMF. Kukuy et al. also reviewed 25 FMF patients undergoing renal biopsies for proteinuria of $>0.5 \mathrm{~g} / 24$ hours (7). While 15 patients $(60 \%)$ had amyloid kidney disease, the remaining 10 patients had other types of nephropathy, including focal glomerulonephritis $(n=5)$, focal segmental glomerular sclerosis $(n=1)$, IgA nephropathy $(n=1)$, focal glomerulonephritis with features of thrombotic microangiopathy $(n=1)$ and focal interstitial nephritis $(n=1)$. Two of the patients without amyloid kidney developed end-stage renal disease at $8.5 \pm 2.1$ years after the onset of proteinuria. Only one patient had hypertension. The authors were unable to clarify the relationship between FMF and non-amyloid 
kidney disease, including nephrosclerosis.

Aksu et al. suggested that FMF patients might have an increased risk of developing inflammatory vascular diseases (vasculitides), such as HSP, classical polyarteritis and protracted febrile myalgia, based on the following hypothesis (10). Increased serum levels of various pro-inflammatory cytokines, including interleukin (IL)-1b $\beta$, IL-6, IL-18, IL-33 and INF- $\gamma$, lead to endothelial cell dysfunction, which seems to be important for the development of vasculitis in FMF patients. IL-1 $\beta$ is the most prominent of these cytokines, and extremely high IL-1b levels may favor the onset of vasculitis in FMF patients. MEFV gene mutations contribute to the occurrence of vasculitis in at least some FMF patients, possibly by increasing the levels of pro-inflammatory cytokines.

Duewell et al. reported that cholesterol crystals can induce inflammation by stimulating the caspase-1-activating NLRP3 (NOD-like receptor family, pyrin domain containing 3 ) inflammasome, resulting in the cleavage and secretion of cytokines from the IL-1 family (11). Vilaysane et al. reported that NLRP3 inflammasome-dependent cytokines, such as IL-1 $\beta$ and IL-18, promote renal inflammation and contribute to the progression of chronic kidney disease in mice (12). Accordingly, research has been commenced into the relationship between atherosclerosis and autoimmune inflammatory diseases, including FMF.

Hyalinotic stenosis of the afferent arterioles due to longstanding hypertension may lead to the release of renin from the juxtaglomerular apparatus (JGA), thus resulting in malignant nephrosclerosis (8). Performing MEFV gene analyses in patients with malignant nephrosclerosis may help to clarify the relationship between this renal condition and autoimmune inflammatory diseases, such as FMF.

In conclusion, a 37-year-old Japanese man was admitted for an evaluation of renal dysfunction and hypertension. The patient had a history of episodes of recurrent abdominal pain with periodic fevers, and a renal biopsy showed malignant nephrosclerosis with onion skin lesions of the arterioles. In addition, a MEFV gene analysis showed that he was homozygous for the E148Q polymorphism and heterozygous for the L110P polymorphism (both affecting exon 2), although he had none of the exon 10 mutations (M694V, M694I, M680I, V726A, etc.) known to be closely related to AA-amyloidosis, which may explain his negativity for AAamyloidosis despite the elevation of both the CRP and SAA levels. The present case emphasizes that patients with FMF can develop renal histological changes consistent with malignant nephrosclerosis and may progress to end-stage renal failure, likely due to the persistent elevation of the levels of NLRP3 inflammasome-dependent cytokines, such as IL-1 $\beta$, if the disease is not diagnosed and treated appropriately.

The authors state that they have no Conflict of Interest (COI).

\section{Acknowledgement}

This study was funded by the Okinaka Memorial Institute for Medical Research.

\section{References}

1. Livneh A, Langevitz P, Zemer D, et al. Criteria for the diagnosis of familial Mediterranean fever. Arthritis Rheum 40: 1879-1885, 1997.

2. Migita K, Uehara R, Nakamura Y, et al. Familial Mediterranean fever in Japan. Medicine (Baltimore) 91: 337-343, 2012.

3. Tomiyama N, Oshiro S, Higashiuesato $\mathrm{Y}$, et al. End-stage renal disease associated with familial Mediterranean fever. Intern Med 41: 221-224, 2002.

4. Nakamura T, Migita K, Ando Y, Takaoka H, Suzushima H, Shiraishi N. Amyloid A amyloidosis in a Japanese patient with familial Mediterranean fever associated with homozygosity for the pyrin variant M694I/M694I. Mod Rheumatol 24: 349-352, 2012.

5. Migita K, Agematsu K, Yazaki M, et al. Familial Mediterranean fever: genotype-phenotype correlations in Japanese patients. Medicine (Baltimore) 93: 158-164, 2014.

6. Said R, Hamzeh Y, Said S, Tarawneh M, al-Khateeb M. Spectrum of renal involvement in familial Mediterranean fever. Kidney Int 41: 414-419, 1992.

7. Kukuy O, Livneh A, Ben-David A, et al. Familial Mediterranean fever (FMF) with proteinuria: clinical features, histology, predictors, and prognosis in a cohort of 25 patients. J Rheumatol 40: 2083-2087, 2013.

8. Nonaka K, Ubara Y, Sumida K, et al. Clinical and pathological evaluation of hypertensive emergency-related nephropathy. Intern Med 52: 45-53, 2013.

9. Tsuchiya-Suzuki A, Yazaki M, Nakamura A, et al. Clinical and genetic features of familial Mediterranean fever in Japan. J Rheumatol 36: 1671-1676, 2009.

10. Aksu K, Keser G. Coexistence of vasculitides with familial Mediterranean fever. Rheumatol Int 31: 1263-1274, 2011.

11. Duewell P, Kono H, Rayner KJ, et al. NLRP3 inflammasomes are required for atherogenesis and activated by cholesterol crystals. Nature 464: 1357-1361, 2010.

12. Vilaysane A, Chun J, Seamone ME, et al. The NLRP3 inflammasome promotes renal inflammation and contributes to CKD. J Am Soc Nephrol 21: 1732-1744, 2010.

(C) 2015 The Japanese Society of Internal Medicine http://www.naika.or.jp/imonline/index.html 